\title{
Synthesis, Biological Evaluation, Docking and QSAR Studies of Some Novel Naphthalimide Dithiocarbamate Analogs as Antitumor and Anti- Inflammatory Agents
}

Maram H Zahra ${ }^{1,2}$, Amany M.A. Osman ${ }^{1}$, Hussein Agwa ${ }^{1}$, Neha Nair$^{2}$, Anna Sanchez Calle ${ }^{2}$, Laura Hurley ${ }^{3}$, Doaa Farag $^{4 *}$, Tomonari Kasai ${ }^{2 *}$ Masaharu Seno² and Magdy Zahran ${ }^{1 *}$

${ }^{1}$ Department of Chemistry, Faculty of Science, Menoufia University, Shebin El-Koam, Egypt

${ }^{2}$ Laboratory of Nano-Biotechnology, Department of Medical Bioengineering Science, Graduate School of Natural Science and Biotechnology, Okayama University, Kitaku, Okayama, Japan

${ }^{3}$ Karmanos Cancer Institute, Wayne State University, Detroit, MI, USA

${ }^{4}$ Pharmaceutical Chemistry Department, Faculty of Pharmacy, Misr International University, Cairo, Egypt

\begin{abstract}
A series of novel naphthalimide dithiocarbamate $\mathbf{4 a - f , ~ 5 a - f ~ w e r e ~ e f f i c i e n t l y ~ s y n t h e s i z e d ~ v i a ~ i n t r o d u c e ~ d i t h i o c a r b a m a t e ~ a n d ~}$ dithioate side chain onto the naphthalic anhydride core. The structures of the synthesized analogs were elucidated by spectroscopic methods, including IR, ${ }^{1} \mathrm{H}$ and ${ }^{13} \mathrm{C}$ NMR, and (ESIHRMS) techniques. The anti-cancer activities of the generated naphthalimide derivatives $\mathbf{4 c}, \mathbf{4 d}, \mathbf{4 e}, \mathbf{4 f}$, and $\mathbf{5 d}$ were evaluated against 21 tumour cell lines; inculding 10 tumor subpanels using MTT assay. Analogue $4 \mathrm{c}$ offer antitumor activity with an $\mathrm{IC}_{50}$ of $10.54 \mu \mathrm{M}$ against SKBR3 breast cancer cells. Compound $\mathbf{4 d}$ showed varying degrees of antitumor activities towards several tumour cell lines ranging from 21.1 to $71.7 \mu \mathrm{M}$. In addition to the antitumor activities; the synthesized compounds were evaluated for their in vitro anti-inflammatory activity. Compounds $\mathbf{4 c}$ and $\mathbf{4 d}$ revealed potent anti-inflammatory properties in comparison with the reference drug celecoxib. Molecular docking studies provided complementary theoretical support for experimental biological data.
\end{abstract}

Keywords: Naphthalimide; Dithiocarbamates; Dithioates; Anticancer; Anti-inflammatory

\section{Introduction}

The development of new classes of chemotherapeutic agents to treat cancer patients remains an important goal of the medical community worldwide. The International Agency for Research on Cancer reported that the global cancer burden rose to 14.1 million new cases with 8.2 million cancer deaths in 2012, and the annual incidence of new cancer cases is estimated to increase to 19.3 million by 2050 [1]. During the past few years, cyclooxygenases (COX-1 and COX-2) were introduced as novel targets for cancer treatment [2]. COX-2 is an effective approach for the prevention or treatment of various types of cancers such as colon, prostate, and breast [3]. Anticancer drugs that target DNA are one of the most effective agents for cancer therapy [4]. DNA intercalating agents are characterized by the presence of a tricyclic or tetracylic annelated, planar, aromatic ring that intercalates between nucleic acid bases and one or two flexible amino side chains that enhance DNA binding affinity via electrostatic or hydrophobic interactions [5]. Among the agents directly intercalating with DNA; naphthalimides which belong to the cyclic imides class represent an important moiety in the antitumor drug design concept [6]. Several biological effects suggest a potential pharmaceutical use of cyclic imides, such as antinociceptive [7], anti-inflammatory [8], antimicrobial [9], and potential antitumor agents against different cancer cell lines $[10,11]$. Historically naphthalimide $[1 H$-benz [de] isoquinoline-1, 3-(2H)-diones] is considered as one of the simplest poly cyclic amides from a famous class of intercalating agents consisting of a flat, generally $\pi$-deficient aromatic or heteroaromatic system which bind to DNA by insertion between base pairs of the double helix [12-14]. The cytotoxic properties of several $N$-substituted naphthalimides are well documented to possess significant anticancer activity [15,16]. Naphthalimide derivatives exhibit their cytostatic activity through DNA intercalation, which causes enzymatic blockade and reading errors during the replication process, inhibiting both RNA and DNA synthesis and generating a multitude of reactive intermediates that result in DNA photocleavage $[17,18]$. The role of the sulfur atom in improving antitumor activities has been reported. Previous studies showed that especially sulfur-containing derivatives exhibited promising antiproliferative efficiency towards several tumor cell lines $[19,20]$. Dithiocarbamates have been attracting considerable interest because of their diverse activities [21-24] and in the treatment of cancer $[25,26]$. Our group recently reported the synthesis of a novel thalidomide-containing dithiocarbamate moiety; several derivatives exhibited potent anti-cancer activity [27,28]. Based on the research for potential antitumor agents, the introduction of alkylamino groups into some pharmacophores can enhance their antitumor activity [29]. In view of this data; this study was carried out to discover novel antitumor agents based on naphthalimide skeleton.

Here we designed, synthesized and characterized a series of novel naphthalimide analogs by conjugating various dithiocarbamate and dithioate moieties to DNA- intercalating naphthalimide ring system (Figure 1). We subsequently evaluated their antitumor activities; fluorescence quenching assays were employed to determine their DNA-binding activity in addition to anti-inflammatory properties.

*Corresponding authors: Tomonari Kasai, Department of Medical Bioengineering Science, Okayama University, Kitaku, Okayama, Japan, Tel: +81862518265 E-mail: t-kasai@cc.okayama-u.ac.jp

Magdy Zahran, Faculty of Science, Menoufia University Shebin El-Koam 2323 Egypt, Fax: +2024159770; E-mail: magdyzahran53@gmail.com

Doaa Farag, Pharmaceutical Chemistry Department, Faculty of Pharmacy, Mis International University, Cairo, Egypt, Tel: +201005667779; Fax: +2024772038 E-mail: doaa.boshra@miuegypt.edu.eg

Received November 24, 2016; Accepted December 07, 2016; Published December 12, 2016

Citation: Zahra M, Osman A, Agwa H, Nair N, Sanchez A, et al. (2016) Synthesis Biological Evaluation, Docking and QSAR Studies of Some Novel Naphthalimide Dithiocarbamate Analogs as Antitumor and Anti-Inflammatory Agents. Med Chem (Los Angeles) 6: 694-703. doi:10.4172/2161-0444.1000417

Copyright: ( 2016 Zahra M, et al. This is an open-access article distributed under the terms of the Creative Commons Attribution License, which permits unrestricted use, distribution, and reproduction in any medium, provided the original author and source are credited. 
Citation: Zahra M, Osman A, Agwa H, Nair N, Sanchez A, et al. (2016) Synthesis, Biological Evaluation, Docking and QSAR Studies of Some Novel Naphthalimide Dithiocarbamate Analogs as Antitumor and Anti-Inflammatory Agents. Med Chem (Los Angeles) 6: 694-703. doi:10.4172/2161-0444.1000417

\section{Experimental Section}

\section{Chemistry}

All chemicals and reagents are commercially available and were used directly without further purification. The progress of the synthesis reactions were monitored using analytical silica gel TLC plates $60 \mathrm{~F}_{254}$ (Merck) and visualized using UV light. The ${ }^{1} \mathrm{H}$ and ${ }^{13} \mathrm{C}$ NMR spectra associated with the synthesized compounds were determined using a Bruker (spectrometer and $101 \mathrm{MHz}$ for ${ }^{13} \mathrm{C}$ ) at $400 \mathrm{MHz}$ and $101 \mathrm{MHz}$, respectively. TMS was used as an internal standard for ${ }^{1} \mathrm{H}$ NMR. To evaluate ion mass, the $\left[\mathrm{MH}^{+}\right]$or $\left[\mathrm{MNa}^{+}\right]$ion was calibrated with $\mathrm{NaI}$ (University of Southern Denmark, Denmark). Infrared (IR) spectra $(\mathrm{KBr})$ were recorded using a Pye-Unicam Sp-883 Perkins-Elmer spectrometer (Micro-analytical Laboratory, Faculty of Science, Cairo University). Melting points were determined with an Electro thermal melting point apparatus, and are uncorrected.

\section{Preparation of naphthalimide dithiocarbamates and dithioate derivatives (4a-f and $5 a-f)$}

Appropriate amine $(1 \mathrm{mmol})$ use in this study was added to an ethanolic solution of $\mathrm{KOH}(1 \mathrm{mmol} / 10 \mathrm{~mL})$. The mixture was cooled in ice bath and $\mathrm{CS}_{2}(10 \mathrm{mmol})$ was added drop wise with stirring. Further agitation of the reaction mixture thus obtained for $1 \mathrm{~h}$ at room temperature then, the solvent was evaporated under reduced pressure, followed by consequent addition of dry ether until precipitation reached completion. Then the precipitate was filtered, washed with dry ether and dried to afford dithiocarbamate and dithioate derivatives with high purity. Then, a solution of the appropriate dithiocarbamates and dithioates $(2 \mathrm{mmol})$ in DMF $(5 \mathrm{~mL})$ was added to compounds 4 and $5(1 \mathrm{mmol})$. The mixture was stirred and heated at $70-80^{\circ} \mathrm{C}$ for 2 h. After cooling the solution was poured into cooled distilled water $(50$ $\mathrm{mL})$. The precipitate was collected by filtration, washed with distilled water and dried. The crude product was purified by crystallization from ethanol $(30 \mathrm{~mL})$. The structures of amines applied in this reaction protocol and the newly synthesized compounds are given in Table 1 and see Supplementary File.

2-(1,3-dioxo-1 H-benzo[de] isoquinolin-2(3H)-yl)ethyl methylcarbamodithioate (4a): Yellow solid; mp. $190-192^{\circ} \mathrm{C}$, Yield $76 \%$. IR (KBr) 3265, 2958, 2936, 1696, 1650, 1587, 1338, $1233 \mathrm{~cm}^{-1} .{ }^{1} \mathrm{H}$ NMR (DMSO- $\left.d_{6}\right) \delta 2.97\left(\mathrm{~d}, 3 \mathrm{H}, \mathrm{CH}_{3}\right), 3.55\left(\mathrm{t}, 2 \mathrm{H},\left(\mathrm{NCH}_{2} \mathrm{CH}_{2} \mathrm{~S}\right), 4.34(\mathrm{t}\right.$, $\left.2 \mathrm{H}, \mathrm{NCH}_{2} \mathrm{CH}_{2} \mathrm{~S}\right), 7.87\left(\mathrm{t}, 2 \mathrm{H}, \mathrm{H}_{\text {arom }}\right), 8.44-8.51\left(\mathrm{~m}, 4 \mathrm{H}, \mathrm{H}_{\text {arom }}\right), 9.91(\mathrm{br}$ s, $1 \mathrm{H}, \mathrm{NH}) .{ }^{13} \mathrm{C}$ NMR (DMSO-d $\left.{ }_{6}\right) \delta 32.17\left(\mathrm{CH}_{3}\right), 33.78\left(\mathrm{NCH}_{2} \mathrm{CH}_{2} \mathrm{~S}\right)$, $38.74\left(\mathrm{NCH}_{2} \mathrm{CH}_{2} \mathrm{~S}\right), 122.06\left(\mathrm{C}_{8 \mathrm{a}}\right), 127.35\left(\mathrm{C}_{3}, \mathrm{C}_{6}\right), 127.47\left(\mathrm{C}_{1} \mathrm{C}_{8}\right), 130.96$ $\left(\mathrm{C}_{4}, \mathrm{C}_{5}\right), 131.39\left(\mathrm{C}_{4 \mathrm{a}}\right), 134.52\left(\mathrm{C}_{2}, \mathrm{C}_{7}\right), 163.52(2 \times \mathrm{C}=\mathrm{O}), 194.82(\mathrm{C}=$ S). HRMS (ESI) $\mathrm{m} / \mathrm{z}$ Calcd for $\mathrm{C}_{16} \mathrm{H}_{14} \mathrm{~N}_{2} \mathrm{O}_{2} \mathrm{~S}_{2} \mathrm{Na}^{+}[\mathrm{M}+\mathrm{Na}]^{+} 353.0389$. Found 353.0401 .

2-(1,3-dioxo- $1 \mathrm{H}$-benzo[de] isoquinolin-2(3H)-yl)ethyl dimethylcarbamodithioate (4b): Pale yellow solid; mp. $189-190^{\circ} \mathrm{C}$, Yield 69\%. IR (KBr) 2966, 2920, 1698, 1655, 1587, 1378, $1251 \mathrm{~cm}^{-1}$. ${ }^{1} \mathrm{H}$ NMR (DMSO-d $) \delta 3.31\left(\mathrm{~s}, 3 \mathrm{H}, \mathrm{CH}_{3}\right), 3.42\left(\mathrm{~s}, 3 \mathrm{H}, \mathrm{CH}_{3}\right), 3.60(\mathrm{t}$, $\left.2 \mathrm{H}, \mathrm{NCH}_{2} \mathrm{CH}_{2} \mathrm{~S}\right), 4.38$ (t, $2 \mathrm{H}, \mathrm{NCH}_{2} \mathrm{CH}_{2} \mathrm{~S}$ ), 7.88 (t, $2 \mathrm{H}, \mathrm{H}_{\text {arom }}$ ), 8.45-



Figure 1: The design strategy of naphthalimide-dithiocarbamates. $8.51\left(\mathrm{~m}, 4 \mathrm{H}, \mathrm{H}_{\text {arom }}\right) \cdot{ }^{13} \mathrm{C}$ NMR (DMSO- $\left.d_{6}\right) \delta 34.48\left(\mathrm{NCH}_{2} \mathrm{CH}_{2} \mathrm{~S}\right), 38.43$ $\left(\mathrm{NCH}_{2} \mathrm{CH}_{2} \mathrm{~S}\right), 41.37,44.96\left(2 \times \mathrm{CH}_{3}\right), 122.02\left(\mathrm{C}_{8 \mathrm{a}}\right), 127.23\left(\mathrm{C}_{3}, \mathrm{C}_{6}\right)$, $127.43\left(\mathrm{C}_{1} \mathrm{C}_{8}\right), 130.79\left(\mathrm{C}_{4}, \mathrm{C}_{5}\right), 131.29\left(\mathrm{C}_{42}\right), 134.37\left(\mathrm{C}_{2}, \mathrm{C}_{7}\right), 163.39(2$ $\times \mathrm{C}=\mathrm{O}), 194.57(\mathrm{C}=\mathrm{S})$. HRMS (ESI) $\mathrm{m} / \mathrm{z}$ Calcd for $\mathrm{C}_{17} \mathrm{H}_{16} \mathrm{~N}_{2} \mathrm{O}_{2} \mathrm{~S}_{2} \mathrm{Na}^{+}$ $[\mathrm{M}+\mathrm{Na}]^{+}$367.0545. Found 367.0530.

2-(1,3-dioxo-1 $H$-benzo[de]isoquinolin-2 $(3 \mathrm{H})$-yl)ethyl hexadecylcarbamo-dithioate (4c): White solid; $\mathrm{mp} \quad 138-140^{\circ} \mathrm{C}$, Yield 76\%. IR (KBr) 3307, 2955, 2915, 1849, 1699, 1649, 1574, 1361, $1233 \mathrm{~cm}^{-1} .{ }^{1} \mathrm{H}$ NMR (DMSO- $\left.d_{6}\right) \delta 0.84\left(\mathrm{t}, 3 \mathrm{H}, \mathrm{CH}_{3}\right), 1.21-1.33(\mathrm{~m}$, $26 \mathrm{H}$, hexdec. $\left.\mathrm{C}_{3-15}-\mathrm{H}\right), 1.43-1.47\left(\mathrm{~m}, 2 \mathrm{H}\right.$, hexdec. $\left.\mathrm{C}_{2}-\mathrm{H}\right), 3.48(\mathrm{t}, 2 \mathrm{H}$, hexdec. $\left.\mathrm{C}_{1}-\mathrm{H}\right), 3.56\left(\mathrm{t}, 2 \mathrm{H}, \mathrm{NCH}_{2} \mathrm{CH}_{2} \mathrm{~S}\right), 4.34\left(\mathrm{t}, 2 \mathrm{H}, \mathrm{NCH}_{2} \mathrm{CH}_{2} \mathrm{~S}\right)$, $7.88\left(\mathrm{t}, 2 \mathrm{H}, \mathrm{H}_{\text {arom }}\right), 8.44-8.51\left(\mathrm{~m}, 4 \mathrm{H}, \mathrm{H}_{\text {arom }}\right), 9.90($ br s, $1 \mathrm{H}, \mathrm{NH}) ;{ }^{13} \mathrm{C}$ NMR (DMSO-d $) \delta 13.97\left(\mathrm{CH}_{3}\right), 22.11$ (hexdec. $\left.\mathrm{C}_{15}\right), 26.28$ (hexdec. $\mathrm{C}_{3}$ ), 28.62, 28.70, 28.89 (hexdec. $\mathrm{C}_{2}, \mathrm{C}_{4}, \mathrm{C}_{13}$ ), 29.01-29.04 (hexdec. $\left.\mathrm{C}_{5-12}, \mathrm{C}_{14}\right), 31.30\left(\mathrm{NCH}_{2} \mathrm{CH}_{2} \mathrm{~S}\right), 31.93\left(\mathrm{NCH}_{2} \mathrm{CH}_{2} \mathrm{~S}\right), 46.67$ (hexdec. $\left.\mathrm{C}_{1}\right)$, $122.06,127.26,127.48,130.84,131.35,134.42$ (aryl), $163.43(2 \times \mathrm{C}=$ O), $194.86(\mathrm{C}=\mathrm{S})$. HRMS (ESI) $\mathrm{m} / \mathrm{z}$ Calcd for $\mathrm{C}_{31} \mathrm{H}_{45} \mathrm{~N}_{2} \mathrm{O}_{2} \mathrm{~S}_{2}{ }^{+}[\mathrm{M}+\mathrm{H}]^{+}$ 541.2917 .Found: 541.2907.

2-(1,3-dioxo-1 H-benzo[de] isoquinolin-2(3H)-yl)ethyl 1,4'-bipiperidine-1'-carbodithioate (4d): Yellow solid; $\mathrm{mp} 133-135^{\circ} \mathrm{C}$, Yield 58\%. IR (KBr) 2934, 2852, 1698, 1659, 1590, 1339, $1234 \mathrm{~cm}^{-1} .{ }^{1} \mathrm{H}$ NMR $\left(\mathrm{CDCl}_{3}\right) \delta 1.45-1.46\left(\mathrm{~m}, 2 \mathrm{H}\right.$, pip. $\left.\mathrm{C}_{3}-\mathrm{H}\right), 1.60\left(\mathrm{~m}, 6 \mathrm{H}\right.$, pip. $\mathrm{C}_{3}$ ', ${ }_{4}{ }^{\prime}{ }_{5}$ - $\left.-\mathrm{H}\right), 1.86-1.89\left(\mathrm{~m}, 2 \mathrm{H}\right.$, pip. $\left.\mathrm{C}_{5}-\mathrm{H}\right), 2.52-2.63\left(\mathrm{~m}, 5 \mathrm{H}\right.$, pip. $\mathrm{C}_{2}{ }^{\prime}{ }_{6}{ }^{-}-\mathrm{H}$, pip. $\mathrm{C}_{4}-\mathrm{H}$ ), 3.10 (br s, $2 \mathrm{H}$, pip. $\left.\mathrm{C}_{2}-\mathrm{H}\right), 3.76\left(\mathrm{t}, 2 \mathrm{H}, \mathrm{NCH}_{2} \mathrm{CH}_{2} \mathrm{~S}\right), 4.55-$ $4.56\left(\mathrm{~m}, 3 \mathrm{H}, \mathrm{NCH}_{2} \mathrm{CH}_{2} \mathrm{~S}\right.$, pip. $\left.\mathrm{C}_{6}-\mathrm{H}\right), 5.55$ (br s, $1 \mathrm{H}$, pip. $\left.\mathrm{C}_{6}-\mathrm{H}\right), 7.75(\mathrm{t}$, $\left.2 \mathrm{H}, \mathrm{H}_{\text {arom }}\right), 8.22\left(\mathrm{~d}, 2 \mathrm{H}, J=8 \mathrm{~Hz}, \mathrm{H}_{\text {arom }}\right), 8.60\left(\mathrm{~d}, 2 \mathrm{H}, J=8 \mathrm{~Hz}, \mathrm{H}_{\text {arom }}\right)$. ${ }^{13} \mathrm{C} \mathrm{NMR}\left(\mathrm{CDCl}_{3}\right) \delta 24.60$ (pip. $\mathrm{C}_{3}{ }^{\text {arom }} \mathrm{C}_{4}, \mathrm{C}_{5}{ }^{\prime}$ ), 26.21 (pip. $\left.\mathrm{C}_{3} \mathrm{C}_{5}\right), 34.89$ $\left(\mathrm{NCH}_{2} \mathrm{CH}_{2} \mathrm{~S}\right), 38.86\left(\mathrm{NCH}_{2} \mathrm{CH}_{2} \mathrm{~S}\right), 50.18$ (pip. $\left.\mathrm{C}_{2}, \mathrm{C}_{6} \mathrm{C}_{2}{ }^{\prime} \mathrm{C}_{6}{ }^{\prime}\right), 62.05$ (pip. $\mathrm{C}_{4}$ ), 122.56, 126.90, 128.20, 131.29, 131.32, 133.95 (aryl), 164.07 (2 $\times \mathrm{C}=\mathrm{O}), 195.12(\mathrm{C}=\mathrm{S})$. HRMS (ESI) $\mathrm{m} / \mathrm{z}$ Calcd for $\mathrm{C}_{25} \mathrm{H}_{30} \mathrm{~N}_{3} \mathrm{O}_{2} \mathrm{~S}_{2}{ }^{+}[\mathrm{M}$ $+\mathrm{H}]^{+}$468.1774. Found: 468.1768 .

2-(1,3-dioxo-1 H-benzo[de] isoquinolin-2(3H)-yl)ethyl4methylpiperazin-1-ylcarbamodithioate (4e): Pale yellow solid; $\mathrm{mp}$ $179-180^{\circ} \mathrm{C}$, Yield $60 \%$. IR (KBr) 3433, 2922, 2849, 1798, 1698, 1661, $1589,1342,1233 \mathrm{~cm}^{-1} .{ }^{1} \mathrm{H}$ NMR (DMSO-d $) 2.18\left(\mathrm{~s}, 3 \mathrm{H}, \mathrm{CH}_{3}\right), 2.32(\mathrm{br}$ s, $4 \mathrm{H}$, pip. $\left.\mathrm{C}_{3,5}-\mathrm{H}\right), 3.65$ (t, $\left.2 \mathrm{H}, \mathrm{NCH}_{2} \mathrm{CH}_{2} \mathrm{~S}\right), 3.85$ (br s, $2 \mathrm{H}$, pip. $\mathrm{C}_{2}-\mathrm{H}$ ), 4.15 (br s, $2 \mathrm{H}$, pip. $\left.\mathrm{C}_{6}-\mathrm{H}\right), 4.38\left(\mathrm{t}, 2 \mathrm{H}, \mathrm{NCH}_{2} \mathrm{CH}_{2} \mathrm{~S}\right), 7.86\left(\mathrm{t}, 2 \mathrm{H}, \mathrm{H}_{\text {arom }}\right.$ ), 8.43-8.49 (m, $\left.4 \mathrm{H}, \mathrm{H}_{\text {arom }}\right) .{ }^{13} \mathrm{C}$ NMR (DMSO- $\left.d_{6}\right) \delta 34.08\left(\mathrm{NCH}_{2} \mathrm{CH}_{2} \mathrm{~S}\right)$, $38.55\left(\mathrm{NCH}_{2} \mathrm{CH}_{2} \mathrm{~S}\right), 45.03\left(\mathrm{NCH}_{3}\right), 53.84$ (pip. $\left.\mathrm{C}_{2}, \mathrm{C}_{6}, \mathrm{C}_{3}, \mathrm{C}_{5}\right), 122.06$, 127.31, 127.50, 130.89, 131.36, $134.46(\operatorname{aryl}), 163.48(2 \times \mathrm{C}=\mathrm{O}), 193.29$ $(\mathrm{C}=\mathrm{S})$. HRMS (ESI) $\mathrm{m} / \mathrm{z}$ Calcd for $\mathrm{C}_{20} \mathrm{H}_{23} \mathrm{~N}_{4} \mathrm{O}_{2} \mathrm{~S}_{2} \mathrm{Na}^{+}[\mathrm{M}+\mathrm{Na}+\mathrm{H}]^{+}$ 438.1155. Found: 438.1148.

2-(1,3-dioxo-1 H-benzo[de] isoquinolin-2(3H)-yl)ethyl4-(2hydroxyethyl)piperazine-1-carbodithioate (4f): Pale yellow solid; mp $152-154^{\circ} \mathrm{C}$, Yield $82 \%$. IR (KBr) 3446, 2934, 2877, 2811, 1697, 1659, 1590, 1341, $1226 \mathrm{~cm}^{-1}$. ${ }^{1} \mathrm{H}$ NMR (DMSO-d) $2.38-2.43(\mathrm{~m}, 6 \mathrm{H}$, $\mathrm{NCH}_{2} \mathrm{CH}_{2} \mathrm{OH}$, pip. $\left.\mathrm{C}_{35}-\mathrm{H}\right), 3.47-3.52\left(\mathrm{~m}, 2 \mathrm{H}, \mathrm{CH}_{2} \mathrm{OH}\right), 3.65(\mathrm{t}, 2 \mathrm{H}$, $\mathrm{NCH}_{2} \mathrm{CH}_{2} \mathrm{~S}$ ), 3.85 (br s, $2 \mathrm{H}$, pip. $\mathrm{C}_{2}-\mathrm{H}$ ), 4.14 (br s, $2 \mathrm{H}$, pip. $\mathrm{C}_{6}-\mathrm{H}$ ), 4.37 $\left(\mathrm{t}, 2 \mathrm{H}, \mathrm{NCH}_{2} \mathrm{CH}_{2} \mathrm{~S}\right), 4.46-4.48\left(\mathrm{~m}, 1 \mathrm{H}, \mathrm{CH}_{2} \mathrm{OH}\right), 7.85\left(\mathrm{t}, 2 \mathrm{H}, \mathrm{H}_{\text {arom }}\right)$, 8.42-8.48 (m, $\left.4 \mathrm{H}, \mathrm{H}_{\text {arom }}\right) \cdot{ }^{13} \mathrm{C} \mathrm{NMR}\left(\mathrm{DMSO}_{-} d_{6}\right) \delta 33.95\left(\mathrm{NCH}_{2} \mathrm{CH}_{2} \mathrm{~S}\right)$, $38.47\left(\mathrm{NCH}_{2} \mathrm{CH}_{2} \mathrm{~S}\right), 52.38$ (pip. $\left.\mathrm{C}_{2}, \mathrm{C}_{6}, \mathrm{C}_{3}, \mathrm{C}_{5}\right), 58.42\left(\mathrm{CH}_{2} \mathrm{OH}\right), 59.45$ $\left(\mathrm{NCH}_{2} \mathrm{CH}_{2} \mathrm{OH}\right), 121.97,127.18,127.39,130.76,131.25,134.33$ (aryl), $163.33(2 \times \mathrm{C}=\mathrm{O}), 194.10(\mathrm{C}=\mathrm{S})$. HRMS (ESI) $\mathrm{m} / \mathrm{z}$ Calcd for $\mathrm{C}_{21} \mathrm{H}_{24} \mathrm{~N}_{3} \mathrm{O}_{3} \mathrm{~S}_{2}^{+}[\mathrm{M}+\mathrm{H}]^{+} 430.1254$. Found: 430.1233 .

3 - ( 1 , 3 - dioxo- 1 H - benzo [ de] isoquinolin-2 (3 H ) - yl) propylmethylcarbamodithioate $(5 \mathrm{a})$ : White solid; $\mathrm{mp} .189-191^{\circ} \mathrm{C}$, Yield 59\%. IR (KBr) 3307, 2935, 1694, 1649, 1588, 1346, $1244 \mathrm{~cm}^{-1}$. ${ }^{1} \mathrm{H}$ NMR (DMSO- $\left.d_{6}\right) \delta 1.97\left(\mathrm{t}, 2 \mathrm{H}, \mathrm{NCH}_{2} \mathrm{CH}_{2} \mathrm{CH}_{2} \mathrm{~S}\right), 2.99(\mathrm{~d}, 3 \mathrm{H}$, $\left.\mathrm{CH}_{3}\right), 3.26\left(\mathrm{t}, 2 \mathrm{H},\left(\mathrm{NCH}_{2} \mathrm{CH}_{2} \mathrm{CH}_{2} \mathrm{~S}\right), 4.13\left(\mathrm{t}, 2 \mathrm{H}, \mathrm{NCH}_{2} \mathrm{CH}_{2} \mathrm{CH}_{2} \mathrm{~S}\right)\right.$, 
Citation: Zahra M, Osman A, Agwa H, Nair N, Sanchez A, et al. (2016) Synthesis, Biological Evaluation, Docking and QSAR Studies of Some Novel Naphthalimide Dithiocarbamate Analogs as Antitumor and Anti-Inflammatory Agents. Med Chem (Los Angeles) 6: 694-703. doi:10.4172/2161-0444.1000417

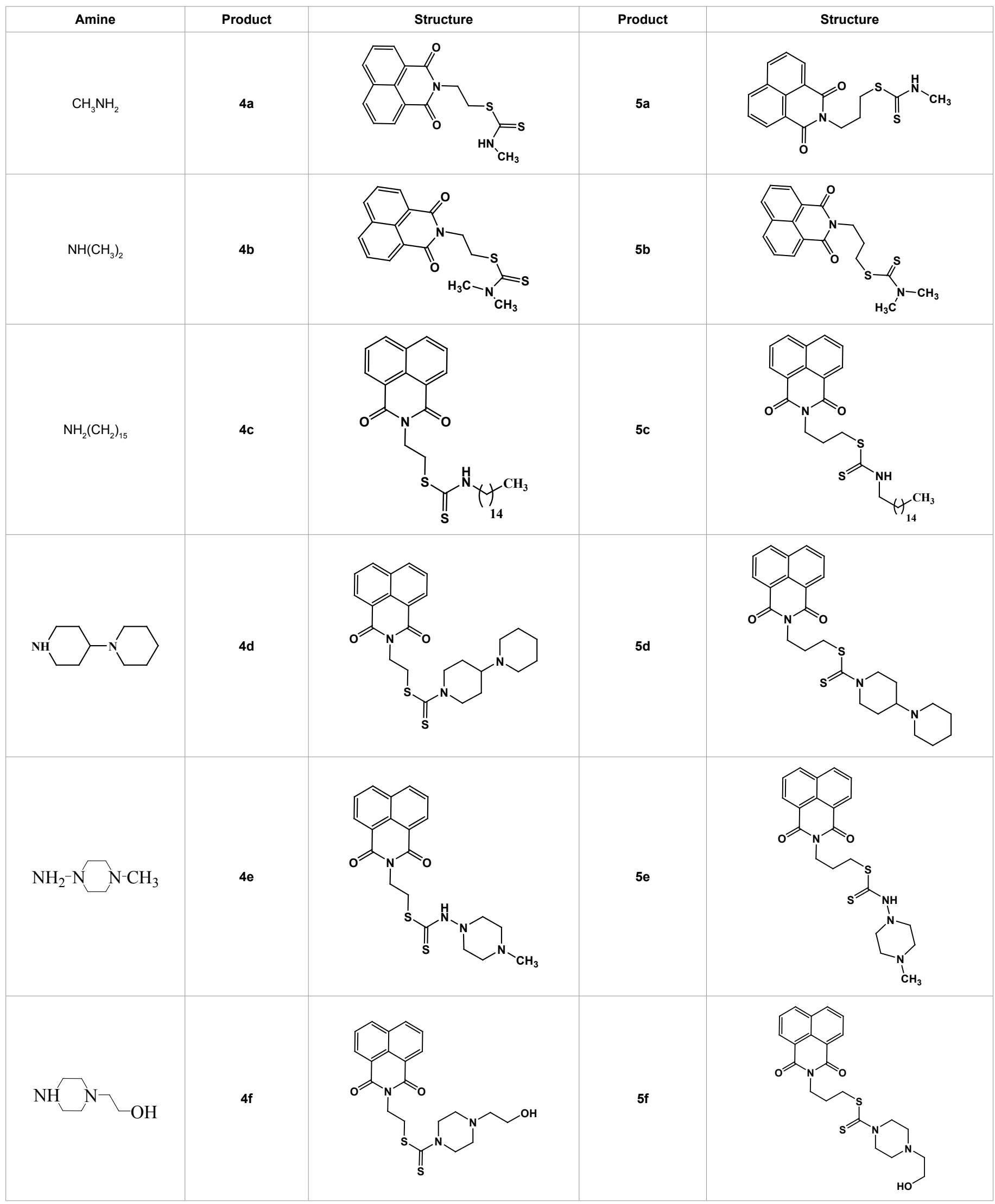

Table 1: Appropriate amine and corresponding naphthalimide dithiocarbamate analogs. 
Citation: Zahra M, Osman A, Agwa H, Nair N, Sanchez A, et al. (2016) Synthesis, Biological Evaluation, Docking and QSAR Studies of Some Novel Naphthalimide Dithiocarbamate Analogs as Antitumor and Anti-Inflammatory Agents. Med Chem (Los Angeles) 6: $694-703$. doi:10.4172/2161-0444.1000417

$7.87\left(\mathrm{t}, 2 \mathrm{H}, \mathrm{H}_{\text {arom }}\right), 8.44-8.49\left(\mathrm{~m}, 4 \mathrm{H}, \mathrm{H}_{\text {arom }}\right), 9.89$ (br s, $\left.1 \mathrm{H}, \mathrm{NH}\right)$. ${ }^{13} \mathrm{C}$ NMR (DMSO- $\left.d_{6}\right) \delta 28.05\left(\mathrm{NCH}_{2} \mathrm{CH}_{2} \mathrm{CH}_{2} \mathrm{~S}\right), 31.74\left(\mathrm{CH}_{3}\right), 33.62$ $\left(\mathrm{NCH}_{2} \mathrm{CH}_{2} \mathrm{CH}_{2} \mathrm{~S}\right), 38.76\left(\mathrm{NCH}_{2} \mathrm{CH}_{2} \mathrm{CH}_{2} \mathrm{~S}\right), 122.13\left(\mathrm{C}_{8 \mathrm{a}}\right), 127.18\left(\mathrm{C}_{3}, \mathrm{C}_{6}\right)$, $127.42\left(\mathrm{C}_{1} \mathrm{C}_{8}\right), 130.68\left(\mathrm{C}_{4}, \mathrm{C}_{5}\right), 131.28\left(\mathrm{C}_{4 \mathrm{a}}\right), 134.25\left(\mathrm{C}_{2}, \mathrm{C}_{7}\right), 163.54(2 \times$ $\mathrm{C}=\mathrm{O}), 196.44(\mathrm{C}=\mathrm{S})$. HRMS (ESI) $\mathrm{m} / \mathrm{z}$ Calcd for $\mathrm{C}_{17} \mathrm{H}_{16} \mathrm{~N}_{2} \mathrm{O}_{2} \mathrm{~S}_{2} \mathrm{Na}^{+}[\mathrm{M}$ $+\mathrm{Na}]^{+}$367.0545. Found 367.0529.

3 - ( 1,3 -dioxo- 1 H-benzo [ de] is oquinolin-2 (3 H ) -yl) propyldimethylcarbamodithioate (5b): Yellow crystal; mp. 173$174^{\circ} \mathrm{C}$, Yield 49\%. IR (KBr) 2924, 1697, 1656, 1590, 1339, $1232 \mathrm{~cm}^{-1}$. ${ }^{1} \mathrm{H}$ NMR (DMSO- $\left.d_{6}\right) \delta 2.00\left(\mathrm{t}, 2 \mathrm{H}, \mathrm{NCH}_{2} \mathrm{CH}_{2} \mathrm{CH}_{2} \mathrm{~S}\right), 3.28(\mathrm{t}, 2 \mathrm{H}$, $\left.\mathrm{NCH}_{2} \mathrm{CH}_{2} \mathrm{CH}_{2} \mathrm{~S}\right), 3.30\left(\mathrm{~s}, 3 \mathrm{H}, \mathrm{CH}_{3}\right), 3.41\left(\mathrm{~s}, 3 \mathrm{H}, \mathrm{CH}_{3}\right), 4.13(\mathrm{t}, 2 \mathrm{H}$, $\left.\mathrm{NCH}_{2} \mathrm{CH}_{2} \mathrm{CH}_{2} \mathrm{~S}\right), 7.86\left(\mathrm{t}, 2 \mathrm{H}, \mathrm{H}_{\text {arom }}\right), 8.42-8.48\left(\mathrm{~m}, 4 \mathrm{H}, \mathrm{H}_{\text {arom }}\right) \cdot{ }^{13} \mathrm{C} \mathrm{NMR}$ $\left(\right.$ DMSO-d $d_{6} \delta 27.49\left(\mathrm{NCH}_{2} \mathrm{CH}_{2} \mathrm{CH}_{2} \mathrm{~S}\right), 34.22\left(\mathrm{NCH}_{2} \mathrm{CH}_{2} \mathrm{CH}_{2} \mathrm{~S}\right), 38.76$ $\left(\mathrm{NCH}_{2} \mathrm{CH}_{2} \mathrm{CH}_{2} \mathrm{~S}\right), 41.12,44.88\left(2 \times \mathrm{CH}_{3}\right), 122.09\left(\mathrm{C}_{82}^{2}\right), 127.15\left(\mathrm{C}_{3}, \mathrm{C}_{6}\right)$, $127.38\left(\mathrm{C}_{1} \mathrm{C}_{8}\right), 130.66\left(\mathrm{C}_{4}, \mathrm{C}_{5}\right), 131.24\left(\mathrm{C}_{4 \mathrm{a}}\right), 134.23\left(\mathrm{C}_{2}, \mathrm{C}_{7}\right), 163.53(2$ $\times \mathrm{C}=\mathrm{O}), 195.20(\mathrm{C}=\mathrm{S})$. HRMS (ESI) $\mathrm{m} / \mathrm{z}$ Calcd for $\mathrm{C}_{18} \mathrm{H}_{18} \mathrm{~N}_{2} \mathrm{O}_{2} \mathrm{~S}_{2} \mathrm{Na}^{+}$ $[\mathrm{M}+\mathrm{Na}]^{+}$381.0702. Found 381.0683.

2 - ( 1,3 -dioxo- $1 \mathrm{H}$-benzo [ de] is oquinolin-2 (3 H ) -yl) propylhexadecylcarbamo-dithioate $(5 \mathrm{c})$ : White solid; $\mathrm{mp} 90-91^{\circ} \mathrm{C}$, Yield 60\%. IR (KBr) 3435, 2920, 2820, 1696, 1659, 1589, 1345, 1232 $\mathrm{cm}^{-1} .{ }^{1} \mathrm{H}$ NMR (DMSO- $\left.d_{6}\right) \delta 0.84\left(\mathrm{t}, 3 \mathrm{H}, \mathrm{CH}_{3}\right), 1.20-1.32(\mathrm{~m}, 26 \mathrm{H}$, hexdec. $\left.\mathrm{C}_{3-15}-\mathrm{H}\right), 1.52,1.61\left(\mathrm{~m}, 2 \mathrm{H}\right.$, hexdec. $\left.\mathrm{C}_{2}-\mathrm{H}\right), 1.95-2.02(\mathrm{~m}, 2 \mathrm{H}$, $\left.\mathrm{NCH}_{2} \mathrm{CH}_{2} \mathrm{CH}_{2} \mathrm{~S}\right), 2.82,3.25\left(\mathrm{t}, 2 \mathrm{H}\right.$, hexdec. $\left.\mathrm{C}_{1}-\mathrm{H}\right), 3.51,3.64(\mathrm{~m}, 2 \mathrm{H}$, $\left.\mathrm{NCH}_{2} \mathrm{CH}_{2} \mathrm{CH}_{2} \mathrm{~S}\right), 4.13\left(\mathrm{t}, 2 \mathrm{H}, \mathrm{NCH}_{2} \mathrm{CH}_{2} \mathrm{CH}_{2} \mathrm{~S}\right), 7.87(\mathrm{t}, 2 \mathrm{H}$, aryl), 8.44$8.50(\mathrm{~m}, 4 \mathrm{H}$, aryl), 9.89 (br s, $1 \mathrm{H}, \mathrm{NH}) .{ }^{13} \mathrm{C}$ NMR (DMSO- $\left.d_{6}\right) \delta 13.95$ $\left(\mathrm{CH}_{3}\right), 22.08$ (hexdec. $\mathrm{C}_{15}$ ), 26.33 (hexdec. $\mathrm{C}_{3}$ ), $27.41\left(\mathrm{NCH}_{2} \mathrm{CH}_{2} \mathrm{CH}_{2} \mathrm{~S}\right)$, 28.11, 28.69, 28.81 (hexdec. $\mathrm{C}_{2}, \mathrm{C}_{4}, \mathrm{C}_{13}$ ), 28.86-29.19 (hexdec. $\mathrm{C}_{5-12}$ ), $31.28\left(\mathrm{C}_{14}\right), 35.72\left(\mathrm{NCH}_{2} \mathrm{CH}_{2} \mathrm{CH}_{2} \mathrm{~S}\right), 44.71\left(\mathrm{NCH}_{2} \mathrm{CH}_{2} \mathrm{CH}_{2} \mathrm{~S}\right), 46.62$ (hexdec. $\mathrm{C}_{1}$ ), 122.07, 127.20, 127.45, 130.70, 131.32, 134.29 (aryl), $163.49(2 \times \mathrm{C}=\mathrm{O}), 195.70(\mathrm{C}=\mathrm{S})$. HRMS (ESI) $\mathrm{m} / \mathrm{z}$ Calcd for $\mathrm{C}_{32} \mathrm{H}_{47}$ $\mathrm{N}_{2} \mathrm{O}_{2} \mathrm{~S}_{2}^{+}[\mathrm{M}+\mathrm{H}]^{+}$555.3073. Found: 555.3066.

2-(1,3-dioxo-1 H-benzo[de] isoquinolin-2 $3 \mathrm{H})$-yl)propyl 1,4'-bipiperidine-1'-carbodithioate (5d): Yellow solid; $\mathrm{mp} 139-140^{\circ} \mathrm{C}$, Yield 85\%. IR (KBr) 2929, 2850, 1695, 1656, 1587, 1341, $1213 \mathrm{~cm}^{-1} .{ }^{1} \mathrm{H}$ NMR (DMSO- $\left.d_{6}\right) \delta 1.32-1.46$ (m, 8H, pip. $\mathrm{C}_{3}-\mathrm{H}$, pip. $\left.\mathrm{C}_{3}{ }^{\prime}{ }_{4}{ }^{\prime}{ }_{5}{ }^{\prime}-\mathrm{H}\right), 1.72-$ $1.74\left(\mathrm{~m}, 2 \mathrm{H}\right.$, pip. $\left.\mathrm{C}_{5}-\mathrm{H}\right), 1.96-2.03\left(\mathrm{~m}, 2 \mathrm{H},\left(\mathrm{NCH}_{2} \mathrm{CH}_{2} \mathrm{CH}_{2} \mathrm{~S}\right), 2.35-2.37\right.$ (m, $\left.4 \mathrm{H}, \mathrm{C}_{2}{ }^{\prime}{ }_{6}{ }^{\prime}-\mathrm{H}\right), 2.52-2.57\left(\mathrm{~m}, 1 \mathrm{H}, \mathrm{C}_{4}-\mathrm{H}\right), 3.10$ (br s, $2 \mathrm{H}$, pip. $\left.\mathrm{C}_{2}-\mathrm{H}\right)$, $3.30\left(\mathrm{t}, 2 \mathrm{H}, \mathrm{NCH}_{2} \mathrm{CH}_{2} \mathrm{~S}\right), 4.12\left(\mathrm{t}, 2 \mathrm{H}, \mathrm{NCH}_{2} \mathrm{CH}_{2} \mathrm{~S}\right.$ ), 4.39 (br s, $1 \mathrm{H}$, pip. $\left.\mathrm{C}_{6}-\mathrm{H}\right), 5.20$ (br s, $1 \mathrm{H}$, pip. $\left.\mathrm{C}_{6}-\mathrm{H}\right), 7.82(\mathrm{t}, 2 \mathrm{H}$, aryl), 8.39-8.44 (m, $4 \mathrm{H}$, aryl). ${ }^{13} \mathrm{C}$ NMR (DMSO- $d_{6}$ ) $\delta 24.43$ (pip. $\mathrm{C}_{4}{ }^{\prime}$ ), 25.98 (pip. $\mathrm{C}_{3}{ }^{\prime}, \mathrm{C}_{5}{ }_{5}, \mathrm{C}_{3,}$ $\left.\mathrm{C}_{5}\right), 27.40\left(\mathrm{NCH}_{2} \mathrm{CH}_{2} \mathrm{CH}_{2} \mathrm{~S}\right), 34.03\left(\mathrm{NCH}_{2} \mathrm{CH}_{2} \mathrm{~S}\right), 38.74\left(\mathrm{NCH}_{2} \mathrm{CH}_{2} \mathrm{~S}\right)$, 49.59 (pip. $\mathrm{C}_{2} \mathrm{C}_{6} \mathrm{C}_{2}{ }^{\prime} \mathrm{C}_{6}{ }^{\prime}$ ), 60.75 (pip. $\mathrm{C}_{4}$ ), 122.01, 127.09, 127.31, 130.60, 131.21, 134.19 (aryl), $163.46(2 \times \mathrm{C}=\mathrm{O}), 194.05(\mathrm{C}=\mathrm{S})$. HRMS (ESI) $\mathrm{m} / \mathrm{z}$ Calcd for $\mathrm{C}_{26} \mathrm{H}_{32} \mathrm{~N}_{3} \mathrm{O}_{2} \mathrm{~S}_{2}^{+}[\mathrm{M}+\mathrm{H}]^{+} 482.1930$. Found: 482.1912.

2-(1,3-dioxo-1 H-benzo[de] isoquinolin-2(3H)-yl)propyl 4-methylpiperazin-1-ylcarbamodithioate (5e): White solid; $\mathrm{mp}$ $150-152^{\circ} \mathrm{C}$, Yield $75 \%$. IR (KBr) 3445, 2961, 2933, 2848, 1700, $1659,1590,1343,1231 \mathrm{~cm}^{-1}$. ${ }^{1} \mathrm{H}$ NMR (DMSO-d $) 1.98-2.05(\mathrm{~m}$, $2 \mathrm{H}, \mathrm{NCH}_{2} \mathrm{CH}_{2} \mathrm{CH}_{2} \mathrm{~S}$ ), 2.17 (s, $3 \mathrm{H}, \mathrm{CH}_{3}$ ), 2.33 (br s, $4 \mathrm{H}$, pip. $\mathrm{C}_{3,5}-\mathrm{H}$ ), $3.31-3.35\left(\mathrm{~m}, 2 \mathrm{H}, \mathrm{NCH}_{2} \mathrm{CH}_{2} \mathrm{~S}\right), 3.84$ (br s, $2 \mathrm{H}$, pip. $\left.\mathrm{C}_{2}-\mathrm{H}\right), 4.15(\mathrm{t}, 4 \mathrm{H}$, $\mathrm{NCH}_{2} \mathrm{CH}_{2} \mathrm{~S}$, pip. $\left.\mathrm{C}_{6}-\mathrm{H}\right), 7.87\left(\mathrm{t}, 2 \mathrm{H}\right.$, aryl), 8.45-8.51 (m, $4 \mathrm{H}$, aryl). ${ }^{13} \mathrm{C}$ $\mathrm{NMR}\left(\mathrm{DMSO}-d_{6}\right) \delta 27.77\left(\mathrm{NCH}_{2} \mathrm{CH}_{2} \mathrm{CH}_{2} \mathrm{~S}\right) 34.09\left(\mathrm{NCH}_{2} \mathrm{CH}_{2} \mathrm{CH}_{2} \mathrm{~S}\right)$, $40.41\left(\mathrm{NCH}_{2} \mathrm{CH}_{2} \mathrm{CH}_{2} \mathrm{~S}\right), 45.23\left(\mathrm{NCH}_{3}\right), 54.11$ (pip. $\left.\mathrm{C}_{2}, \mathrm{C}_{6}, \mathrm{C}_{3}, \mathrm{C}_{5}\right)$, $122.34,127.61,127.71,131.16,131.60,134.72$ (aryl), $164.06(2 \times \mathrm{C}=$ O), $195.51(\mathrm{C}=\mathrm{S})$. HRMS (ESI) $\mathrm{m} / \mathrm{z}$ Calcd for $\mathrm{C}_{21} \mathrm{H}_{25} \mathrm{~N}_{4} \mathrm{O}_{2} \mathrm{~S}_{2}{ }^{+}[\mathrm{M}+\mathrm{H}]^{+}$ 429.1413. Found: 429.1397.

2-(1,3-dioxo-1 H-benzo[de]isoquinolin-2(3H)-yl)propyl 4-(2-hydroxyethyl) piperazine-1-carbodithioate (5f): Pale yellow solid; mp $147-149^{\circ} \mathrm{C}$, Yield $87 \%$. IR (KBr) 3435, 2920, 2820, 1696,
$1659,1589,1345,1232 \mathrm{~cm}^{-1} .{ }^{1} \mathrm{H}$ NMR (DMSO- $\left.d_{6}\right) 1.99-2.03(\mathrm{~m}$, $\left.2 \mathrm{H}, \mathrm{NCH}_{2} \mathrm{CH}_{2} \mathrm{CH}_{2} \mathrm{~S}\right), 2.38-2.44\left(\mathrm{~m}, 6 \mathrm{H}, \mathrm{NCH}_{2} \mathrm{CH}_{2} \mathrm{OH}\right.$, pip. $\left.\mathrm{C}_{3,5}-\mathrm{H}\right)$, 3.31-3.33 (m, 2H, $\left.\mathrm{NCH}_{2} \mathrm{CH}_{2} \mathrm{CH}_{2} \mathrm{~S}\right), 3.47-3.51\left(\mathrm{~m}, 2 \mathrm{H}, \mathrm{CH}_{2} \mathrm{OH}\right)$, 3.83 (br s, $2 \mathrm{H}$, pip. $\left.\mathrm{C}_{2}-\mathrm{H}\right), 4.15\left(\mathrm{t}, 4 \mathrm{H}, \mathrm{NCH}_{2} \mathrm{CH}_{2} \mathrm{CH}_{2} \mathrm{~S}\right.$, pip. $\left.\mathrm{C}_{6}-\mathrm{H}\right)$, $4.46\left(\mathrm{t}, 1 \mathrm{H}, \mathrm{CH}_{2} \mathrm{OH}\right), 7.86(\mathrm{t}, 2 \mathrm{H}, \operatorname{aryl}), 8.44-8.49(\mathrm{~m}, 4 \mathrm{H}$, aryl $) .{ }^{13} \mathrm{C}$ NMR (DMSO-d $\left.d_{6}\right) \delta 27.45\left(\mathrm{NCH}_{2} \mathrm{CH}_{2} \mathrm{CH}_{2} \mathrm{~S}\right), 33.78\left(\mathrm{NCH}_{2} \mathrm{CH}_{2} \mathrm{CH}_{2} \mathrm{~S}\right)$, $38.77\left(\mathrm{NCH}_{2} \mathrm{CH}_{2} \mathrm{CH}_{2} \mathrm{~S}\right), 52.48$ (pip. $\left.\mathrm{C}_{2}, \mathrm{C}_{6}, \mathrm{C}_{3}, \mathrm{C}_{5}\right), 58.45\left(\mathrm{CH}_{2} \mathrm{OH}\right)$, $59.47\left(\mathrm{NCH}_{2} \mathrm{CH}_{2} \mathrm{OH}\right), 122.13,127.20,127.42,130.70,131.29,134.28$ (aryl), $163.58(2 \times \mathrm{C}=\mathrm{O}), 194.87(\mathrm{C}=\mathrm{S})$. HRMS (ESI) $\mathrm{m} / \mathrm{z}$ Calcd for $\mathrm{C}_{22} \mathrm{H}_{26} \mathrm{~N}_{3} \mathrm{O}_{3} \mathrm{~S}_{2}^{+}[\mathrm{M}+\mathrm{H}]^{+} 444.1410$.Found: 444.1389.

\section{Biological activities}

The cytotoxic activity of the naphthalimide analogs $(\mathbf{4 c}, \mathbf{4 d}, \mathbf{4 e}$, 4f, and 5f) was evaluated using a 3-(4, 5-dimethylthiazol-2-yl)-2, 5-diphenyltetrazolium bromide (MTT) (Sigma-Aldrich, St. Louis, MO, USA) cleavage assay; against human breast cancer (MCF-7, T-47D, SKBR3, BT474, MB231 and BT474), pancreas (KLM-1, PK8 and PK59) hepatocellular (HepG2, Huh-7 and PLC/PRF-5), Glioma (A127 and U251-MG), lung (Lu99B), colon carcinoma (HT-29 and CW-2), spleen (PMF-ko14), lymphocyte (MY), Ovarian (SKOV3 and OVK18), and T-cell leukemia (MOLT-4). The compounds that exhibited bad solubility or precipitated in cell culture media were omitted from biological evaluation experiments. The cells were seeded in 96-well plates $\left(5 \times 10^{3}\right.$ cells/well $)$ in DMEM or PPMI supplemented with $10 \%$ FBS. After $24 \mathrm{~h}$ in culture, the analogs were added to the cells at concentrations ranging from 6.25 to $100 \mu \mathrm{M}$ for $72 \mathrm{~h}$. The cells were then exposed to MTT ( $5 \mathrm{mg} / \mathrm{mL}$ in PBS) at a final concentration of $1 \mathrm{mg} / \mathrm{mL}$ for $4 \mathrm{~h}$. Formazan crystals that had formed during the incubation period were dissolved overnight at $37^{\circ} \mathrm{C}$ by adding $10 \%$ SDS supplemented with $0.02 \mathrm{~N} \mathrm{HCl}$. The absorbance at $570 \mathrm{~nm}$ was subsequently measured. The experiments were performed in triplicate; and the results were reported in Table 2.

\section{Cyclooxygenase inhibition assay}

The ability of the test compounds to inhibit ovine COX-1 and COX2 was determined using an enzyme immunoassay (EIA) (kit catalog number 560101, Cayman Chemical, Ann Arbor, MI, USA), according to the manufacturer's instructions for cyclooxygenase inhibition assay [30]. The experiments were performed in triplicate and the results were given in Table 3.

\section{Intercalation studies to ct-DNA}

A solution of compounds $\mathbf{4 c}, \mathbf{4 d}, \mathbf{4 e}, \mathbf{4 f}$, and $\mathbf{5 f}(0.25 \mathrm{~mL})$ in DMSO $\left(10^{-3} \mathrm{M}\right)$ was added to $20 \mathrm{mM}$ Tris- $\mathrm{HCl}$ ( $\left.\mathrm{pH} 7.5\right)$ to a final volume of $5 \mathrm{ml}$. The control group of samples was treated with the compound $(100 \mu \mathrm{M})$ in the absence of calf thymus DNA. The other samples were incubated with DNA $(25,50,100$, or $200 \mu \mathrm{M})$. The reactions were shaken for 3 days at $25^{\circ} \mathrm{C}$ in the dark. Fluorescence intensities were measured using the following parameters: excitation: 405, emission: $460 \mathrm{~nm}$.

\section{Docking methodology}

The five promising synthesized compounds $(\mathbf{4 c}, \mathbf{4 d}, \mathbf{4 e}, \mathbf{4 f}$, and 5f) as well as Celecoxib were exported to Discovery Studio 2.5 and prepared for docking using Prepare Ligands protocol. The Prepare Ligands protocol helps to prepare ligands for input into other protocols, performing tasks such as removing duplicates, enumerating isomers and tautomers, and generating $3 \mathrm{D}$ conformations. The pdb codes used for COX-2 is (3LN1), and for COX-1 is (3KK6), loaded from the Protein Data Bank. These proteins were optimized and prepared through a Prepare Protein protocol, which is one of the general 
Citation: Zahra M, Osman A, Agwa H, Nair N, Sanchez A, et al. (2016) Synthesis, Biological Evaluation, Docking and QSAR Studies of Some Novel Naphthalimide Dithiocarbamate Analogs as Antitumor and Anti-Inflammatory Agents. Med Chem (Los Angeles) 6: 694-703. doi:10.4172/2161-0444.1000417

\begin{tabular}{|c|c|c|c|c|c|}
\hline Cell line & $4 c$ & 4d & $4 e$ & $4 \mathrm{f}$ & $5 d$ \\
\hline \multicolumn{6}{|l|}{ Breast cancer } \\
\hline MCF-7 & $81.18 / 31.38 \pm 0.07$ & $56.69 / 71.72 \pm 0.57$ & $43.67 />100$ & $66.32 / 47.17 \pm 0.14$ & $47.3 />100$ \\
\hline SKBR3 & $84.13 / 10.54 \pm 0.33$ & $75.61 / 38.86 \pm 0.81$ & $48.71 />100$ & $50.67 / 93.19 \pm 0.35$ & $34.04 />100$ \\
\hline BT474 & $34.38 />100$ & $87.95 / 26.57 \pm 0.12$ & $33.15 />100$ & $41.49 />100$ & $81.55 / 38.58 \pm 0.11$ \\
\hline MB231 & $52.4 / 88.75 \pm 0.62$ & $85.97 / 34.31 \pm 0.32$ & $40.49 />100$ & $47.88 />100$ & $84.17 / 39.98 \pm 0.07$ \\
\hline T47D & $19.08 />100$ & $75.78 / 21.1 \pm 0.18$ & $78.29 / 29.13 \pm 0.15$ & $24.58 />100$ & $31.98 />100$ \\
\hline \multicolumn{6}{|c|}{ Pancreas cancer } \\
\hline KLM-1 & $46.68 />100$ & $68.89 / 70.48 \pm 0.48$ & $28.65 />100$ & $16.36 />100$ & $47.92 />100$ \\
\hline PK8 & $39.35 />100$ & $59.92 / 27.36 \pm 3.16$ & $25.17 />100$ & $27.4 />100$ & $>100$ \\
\hline PK59 & $76.51 / 36.52 \pm 0.155$ & $77.58 / 40.35 \pm 0.12$ & $60.48 / 72.22 \pm 0.14$ & $44.52 />100$ & $75.27 / 42.41 \pm 0.06$ \\
\hline \multicolumn{6}{|l|}{ Liver cancer } \\
\hline HepG2 & $55.18 / 65.25 \pm 0.743$ & $87.12 / 35.36 \pm 0.02$ & $30.19 />100$ & $24.72 />100$ & $76.80 / 46.35 \pm 0.11$ \\
\hline Huh-7 & $40.93 />100$ & $80.20 / 25.21 \pm 0.06$ & $32.19 />100$ & $47.37 />100$ & $61.91 / 37.47 \pm 0.06$ \\
\hline PLC/PRF-5 & $34.07 />100$ & $36.97 />100$ & $22.66 />100$ & $11.83 />100$ & $56.02 / 77 \pm 0.02$ \\
\hline \multicolumn{6}{|l|}{ Glioma } \\
\hline U251-MG & $>100$ & $79.29 / 22.19 \pm 0.33$ & $33.87>100$ & $29.74>100$ & $40.12 />100$ \\
\hline A172 & $17.09 />100$ & $46.07 />100$ & $24.71 />100$ & $>100$ & $49.08 />100$ \\
\hline \multicolumn{6}{|l|}{ Lung } \\
\hline Lu99B & $34.75 />100$ & $64.2 / 47.07 \pm 0.26$ & $14.94 />100$ & $>100$ & $49.63 />100$ \\
\hline \multicolumn{6}{|l|}{ Colon } \\
\hline $\mathrm{CW}-2$ & $16.01 />100$ & $79.07 / 41.16 \pm 0.01$ & $>100$ & $33.25 />100$ & $51.42 / 96.51 \pm 0.93$ \\
\hline HT-29 & $85.6 / 25.09 \pm 0.01$ & $70.13 / 35.62 \pm 0.05$ & $55.21 / 46.76 \pm 0.88$ & $61.7 / 33.05 \pm 0.6$ & $72.93 / 38.77 \pm 0.28$ \\
\hline PMF-ko14 & $35.59>100$ & $70.44 / 37.89 \pm 0.09$ & $27.43 />100$ & $74.97 / 31.03 \pm 0.18$ & $45.63 />100$ \\
\hline \multicolumn{6}{|l|}{ Lymphocyte } \\
\hline MY & $52.38 / 51.52 \pm 0.62$ & $60.34 / 29.86 \pm 0.27$ & $36.09 />100$ & $44.07 />100$ & $65.64 / 44.52 \pm 0.19$ \\
\hline \multicolumn{6}{|l|}{ Ovary } \\
\hline SKOV3 & $58.03 / 47.27 \pm 0.17$ & $65.76 / 59.77 \pm 0.1$ & $39.94 />100$ & $45.73 />100$ & $54.16 / 89.12 \pm 0.15$ \\
\hline OVK18 & $78.76 / 38.97 \pm 0.13$ & $87.84 / 49.43 \pm 0.14$ & $15.43 />100$ & $13.8 />100$ & $71.53 / 73.96 \pm 0.06$ \\
\hline \multicolumn{6}{|c|}{ T-cell leukemia } \\
\hline MOLT4 & $82.52 / 28.38 \pm 0.06$ & $73.32 / 35.17 \pm 0.17$ & $24.96 />100$ & $62.71 / 46.34 \pm 0.11$ & $38.09 />100$ \\
\hline
\end{tabular}

Table 2: Percentage growth inhibition $(\mathrm{Gl} \%) / / \mathrm{C}_{50}(\mu \mathrm{M} / \mathrm{ml})$ against selected cancer cell lines.

\begin{tabular}{|c|c|c|}
\hline Compound & $\mathbf{I C}_{50}{ }^{a}, \boldsymbol{\mu M}(\mathbf{C O X}-\mathbf{1})$ & $\mathbf{I C}_{50}{ }^{\mathbf{a}}, \boldsymbol{\mu M}(\mathbf{C O X}-\mathbf{2})$ \\
\hline $\mathbf{4 c}$ & $5.71 \pm 0.23$ & $0.74 \pm 0.15$ \\
\hline $\mathbf{4 d}$ & $4.91 \pm 0.62$ & $0.54 \pm 0.73$ \\
\hline $\mathbf{4 e}$ & $9.62 \pm 0.11$ & $1.98 \pm 0.51$ \\
\hline $\mathbf{4 f}$ & $8.40 \pm 0.92$ & $1.42 \pm 0.65$ \\
\hline $\mathbf{5 d}$ & $11.40 \pm 0.39$ & $3.17 \pm 0.48$ \\
\hline Celecoxib & $7.90 \pm 0.20$ & $1.23 \pm 0.05$ \\
\hline
\end{tabular}

Table 3: In vitro inhibitory effect of COX-1 and COX-2 enzymes.

purpose protocols. The Prepare Protein protocol prepares proteins for input into other protocols, performing tasks such as inserting missing atoms in incomplete residues, modeling missing loop regions, deleting alternate conformations, removing un-needed waters, standardizing atom names, and protonating titratable residues using predicted $\mathrm{pKs}$

\section{Results and Discussion}

\section{Chemistry}

As a further extension of our research for promising antitumor compounds [31,32] and in this endeavor a series of noveldithiocarbamate-naphthalimide hybrids have been prepared (4a-f, 5af) by merging the bridgeheads of various dithiocarbamate and dithioate moieties to naphthalimide core. The novel naphthalimide derivatives were synthesized according to the procedures depicted in Scheme 1. Hydroxyl derivatives $(\mathbf{2}, \mathbf{3})$ were generated by refluxing commercially available 1, 8-naphthalic anhydride (1) with a corresponding amino alcohol [33]. Compounds $\mathbf{2}$ and $\mathbf{3}$ were converted to the corresponding chloro derivatives $(\mathbf{4}, \mathbf{5})$ via our modified methodology using thionyl chloride under ice cooling in DMF [34,35]. The target compounds (4a-f, 5a-f) were obtained by nucleophilic substitution of the chloro group with $\mathrm{RCS}_{2} \mathrm{~K}$ in DMF under thermal conditions $70-80^{\circ} \mathrm{C}$. The structures of amines applied in this reaction protocol are given in Table 1 . The newly synthesized compounds were identified using IR, ${ }^{1} \mathrm{H}$ NMR and ${ }^{13} \mathrm{C}$, and electrospray ionization with high resolution mass spectra (ESI/HRMS). We then evaluated the antitumor activity of the resulting derivatives as well as their anti-inflammatory and DNA binding properties.

\section{Biological evaluation}

In vitro cytotoxic activity: The synthetic derivatives $4 \mathrm{c}, \mathbf{4 d}, \mathbf{4 e}, \mathbf{4 f}$, and 5d were evaluated for their in vitro cytotoxic activity against 21 cell lines by MTT assay and the results are given in Table 2 . From the preliminary screening we concluded that; compound $4 \mathrm{c}$ was found to be sensitive to SKBR3 and MCF-7 with $\mathrm{IC}_{50}$ values in $10.54,31.38 \mu \mathrm{M} /$ $\mathrm{ml}$ respectively. Breast cancer cell lines BT474 and MB231 proved to be sensitive towards compounds $\mathbf{4 d}$ and $\mathbf{5 d}$ with $\mathrm{IC}_{50}$ values $26.57,38.58$, 34.31 and $39.98 \mu \mathrm{M} /$ respectively. Compounds $4 \mathbf{d}$ and $4 \mathrm{e}$ exhibited 
Citation: Zahra M, Osman A, Agwa H, Nair N, Sanchez A, et al. (2016) Synthesis, Biological Evaluation, Docking and QSAR Studies of Some Novel Naphthalimide Dithiocarbamate Analogs as Antitumor and Anti-Inflammatory Agents. Med Chem (Los Angeles) 6: 694-703. doi:10.4172/2161-0444.1000417

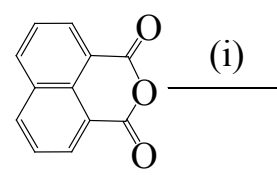

1

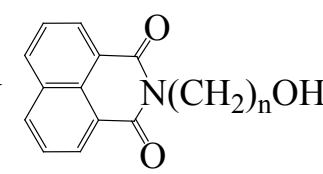

(2) $n=2$

(3) $n=3$ (ii)<smiles>CN1C(=O)C2CCCC3CCC(CC3)C2C1=O</smiles>

(4) $n=2$ (5) $n=3$

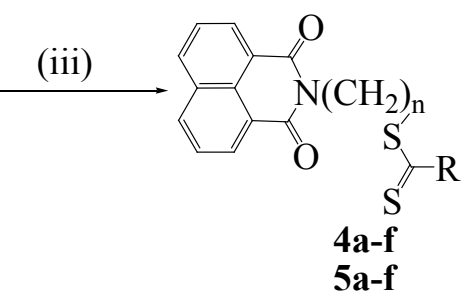

Scheme 1: Reagents and reaction conditions: (i) $\mathrm{H}_{2} \mathrm{~N}\left(\mathrm{CH}_{2}\right)_{n} \mathrm{OH}, \mathrm{EtOH}$, reflux; (ii) thionyl chloride, $0^{\circ} \mathrm{C}, 2 \mathrm{~h}$; (iii) $\mathrm{RCS}{ }_{2} \mathrm{~K}, \mathrm{DMF}, 70-80^{\circ} \mathrm{C}, 2 \mathrm{~h}$.

sensitivity toward breast cancer cell lines T47D with $\mathrm{IC}_{50}$ values of 21.1 and $29.13 \mu \mathrm{M} / \mathrm{ml}$ respectively. Among the pancreatic cancer cell lines, PK59 the order of cytotoxicity exhibited by these derivatives $\left(\mathrm{IC}_{50}\right)$ was $\mathbf{4 c}>\mathbf{4 d}>\mathbf{5 d}>\mathbf{4 e}$; while for PK8 compound $\mathbf{4 d}$ showed good activities with $\mathrm{IC}_{50}$ value $27.36 \mu \mathrm{M} / \mathrm{ml}$. Hepatocellular cancer cell lines HepG2 and Huh-7 proved to be sensitive toward compounds 4d with $\mathrm{IC}_{50}$ values 35.36 and $25.21 \mu \mathrm{M} / \mathrm{ml}$ respectively. Glioblastoma U251MG showed sensitivity only towards compound $\mathbf{4 d}$ with $\mathrm{IC}_{50}$ values $22.19 \mu \mathrm{M} / \mathrm{ml}$. Colon cancer cell lines HT-29 were highly affected by all tested compounds (4c, $\mathbf{4 d}, \mathbf{4 e}, \mathbf{4 f}$, and $\mathbf{5 d}$ ) with $\mathrm{IC}_{50}$ values 25.09, $33.05,46.76,35.62$ and $38.77 \mu \mathrm{M} / \mathrm{ml}$ respectively. Among the evaluated compounds, $\mathbf{4 d}$ and $\mathbf{4 f}$ exhibited sensitivity towards spleen cancer cell line PMF-ko14 with $\mathrm{IC}_{50}$ values 37.89 and $31.03 \mu \mathrm{M} / \mathrm{ml}$ respectively. Lymphocyte cancer cell line MY displayed good activity towards compound 4d with $\mathrm{IC}_{50}$ value $29.86 \mu \mathrm{M} / \mathrm{ml}$. Ovarian cancer cell lines SKOV3 and OVK18 exhibited sensitivity toward Compound $4 \mathbf{c}$ with $\mathrm{IC}_{50}$ values 47.27 and $38.97 \mu \mathrm{M} / \mathrm{ml}$ respectively. Based on the reported data, the stabilization of DNA-drug complexes may be caused by the formation of hydrogen bonds between the amino group and sugar phosphate chain [36]. Compounds $\mathbf{4 c}$, $\mathbf{4 e}$ and $\mathbf{4 f}$ could form similar hydrogen bonds which further block DNA replication; thus exerting their activity. Although compounds $\mathbf{4 d}$ and $\mathbf{5 d}$ do not have a hydrogen bond donor group; during the experimental situation the protonation of the terminal nitrogen in the pipridine group greatly facilitates the DNA intercalation by an initial electrostatic contact with the anionic DNA polymer and this might be the reason for the activity $[13,37,38]$. It is noteworthy that the selective cytotoxicity was possibly due to different membrane crossing ability of the compounds for different cell lines in addition to; the nature of the amino alkyl side chain connected with the chromophore [39]. The preliminary evaluation highlighted compound $4 \mathrm{c}$ as the most potent in vitro cytotoxic agent. Consequently, analog $4 \mathrm{c}$ was selected as a prospective candidate for in vivo studies. Prior to that, with the purpose to enhance the efficiency of the anticancer effect, a drug-delivery system (DDS) based on liposomal encapsulation is currently under development following our pervious publication [40]. Once the optimization of the DDS is achieved, further experimental analysis for the cytotoxicity in normal cell lines will be required in order to assess the possible side effects resulting from the chemotherapeutic effect of this analog. Since this is the first reported paper about the naphthalimide-dithiocarbamate and their antitumor activities further structure optimization is required. To that end, the introduction of various enantiomerically pure amino acids should enhance the potency of the naphthalimides analogs, since recent studies have reported that various drug amino acid conjugates, such as 9-hydroxyellipticinium, anthraquinone, doxorubicin, vinblastin, hydroxymethylacylfulvene, imidazotetrazines, methionine-enkephalin and camptothecins improves the antitumor activity [41].
In vitro cyclooxygenase inhibition assay: It is well known that COX enzymes especially COX-2 are overexpressed in many types of tumor; this strong association make it a valid target for treatment of cancer [41]. In an attempt to investigate the molecular mechanism of the synthesized compound, COX inhibition assay was performed. The ability of compounds $\mathbf{4 c - 4 f}$ and $\mathbf{5 d}$ to inhibit both COX-1 and COX2 was determined and the results are given in Table 3. According to the $\mathrm{IC}_{50}$ data, compounds $\mathbf{4} \mathbf{c}$ and $\mathbf{4 d}$ showed potent activity against both COX subtypes. The results obtained from in vitro cytotoxicity studies were consistent with cyclooxygenase inhibition data. From these results; we could infer that COX inhibition play a key role in the modulation of cytotoxic potencies of Naphthalimide analogs.

\section{Molecular docking study}

The docking study was performed using the Dock Ligands (CDOCKER) protocol, which is one of the Receptor-Ligand Interactions protocols in Acclerys Discovery Studio 2.5. The docking protocol was chosen and validated after comparing results from different docking protocols with the co-crystallized Celecoxib in COX-2 (pdb code 3LN1), and COX-1 (pdb code 3KK6), through alignment of the docked Celecoxib with the co-crystallized one and calculating the RMSD value, which was 0.90 , as well as comparing the binding interactions formed with the receptor's amino acids [42-44]. The docking results are presented in Table 4 and Figure 2. It was found that most of the synthesized compounds and Celecoxib interact with the same binding orientations inside COX-2 active site.

\section{QSAR study}

The QSAR study was performed using Discovery Studio 2.5 Software. The training set for the present QSAR modelling was composed of the 21 compounds with reported COX-2 activity [45] while the test set was composed of $\mathbf{4 c}, \mathbf{4 d}, \mathbf{4 e}, \mathbf{4 f}$, and $\mathbf{5 d}$. The validation for the QSAR model employed was leave one-out cross-validation (internal validation), external validation using Celecoxib, as well as residuals between the predicted and experimental activity of the test set. "Calculate Molecular Properties" module was used for calculating the 2D molecular properties such as AlogP, fingerprints, molecular properties, surface area, volume and the topological descriptors. The partial Least Squares (PLS) model was employed to search for optimal QSAR models that combine high quality binding pharmacophores with other molecular descriptors and being capable of correlating bioactivity variation across the used training set collection. The trials were held while changing the independent properties until the best model with the least variables was obtained. QSAR models were validated employing leave one-out cross-validation, $r^{2}$ (squared correlation coefficient value), and external validation using Celecoxib, as well as residuals between the predicted and experimental activity of the test set; the results are displayed in Table 4 . 
Citation: Zahra M, Osman A, Agwa H, Nair N, Sanchez A, et al. (2016) Synthesis, Biological Evaluation, Docking and QSAR Studies of Some Novel Naphthalimide Dithiocarbamate Analogs as Antitumor and Anti-Inflammatory Agents. Med Chem (Los Angeles) 6: 694-703. doi:10.4172/2161-0444.1000417

\section{QSAR validation}

QSAR models were validated employing leave one-out crossvalidation (internal validation), calculating the residuals between experimental activities and those predicted by the QSAR model, as well as calculating the predicted activity for Celecoxib through running as an external test the compound on the constructed QSAR model using "Calculate Molecular Properties" protocol and selecting the model from the "Other" set. The regression values were as follows: $r=0.956$, $\mathrm{r}^{2}=0.911, \mathrm{r}^{2}$ (adjusted) $=1.064$ and Least-squared error $=0.062$. The experimental activities and those predicted by QSAR studies. Equation 1 represents the best performing QSAR model (Table 5).

Predicted $\mathrm{pIC}_{50}=0.311 *[\mathrm{~A} \log \mathrm{P}]+0.061^{*} \quad[$ Molecular FractionalPolarSurfaceArea] +0.389

It should be noted that the predicted activities by our QSAR models were very close to those experimentally observed, indicating that these models can be safely applied for prediction of more effective hits having the same skeletal framework. Scatter plots of the experimental versus the predicted activity values $\left(\mathrm{pIC}_{50}\right.$ ) according to Equation 1 are presented in Figure 3.

\section{DNA binding study}

The antitumor activity of any compound can be determined by many factors, including cell membrane crossing ability, protein transport, DNA binding ability and drug metabolism [20]. Previous studies have demonstrated that compounds with strong DNAbinding affinity are highly cytotoxic [37]. The fluorescence spectra for compounds $\mathbf{4 c}, \mathbf{4 d}, \mathbf{4 e}, \mathbf{4 f}$, and $\mathbf{5 d}$ are presented in Figure 1. As the compound intercalate DNA, the fluorescence emission intensities decrease as the concentration of DNA increases [46,47]. For all the compounds, fluorescence quenching was decreased with increasing concentrations of DNA (up to $100 \mu \mathrm{M}$ ), and subsequently remained constant. These compounds have the ability to intercalate the DNA helix; forming complex which further blocks DNA replication thus exerting their cytotoxic activities. Our results are consistent with previous studies, which suggested that naphthalimides have DNA intercalating properties [48] (Figure 4).

\section{Conclusion}

Here; we report the synthesis and biological evaluation of

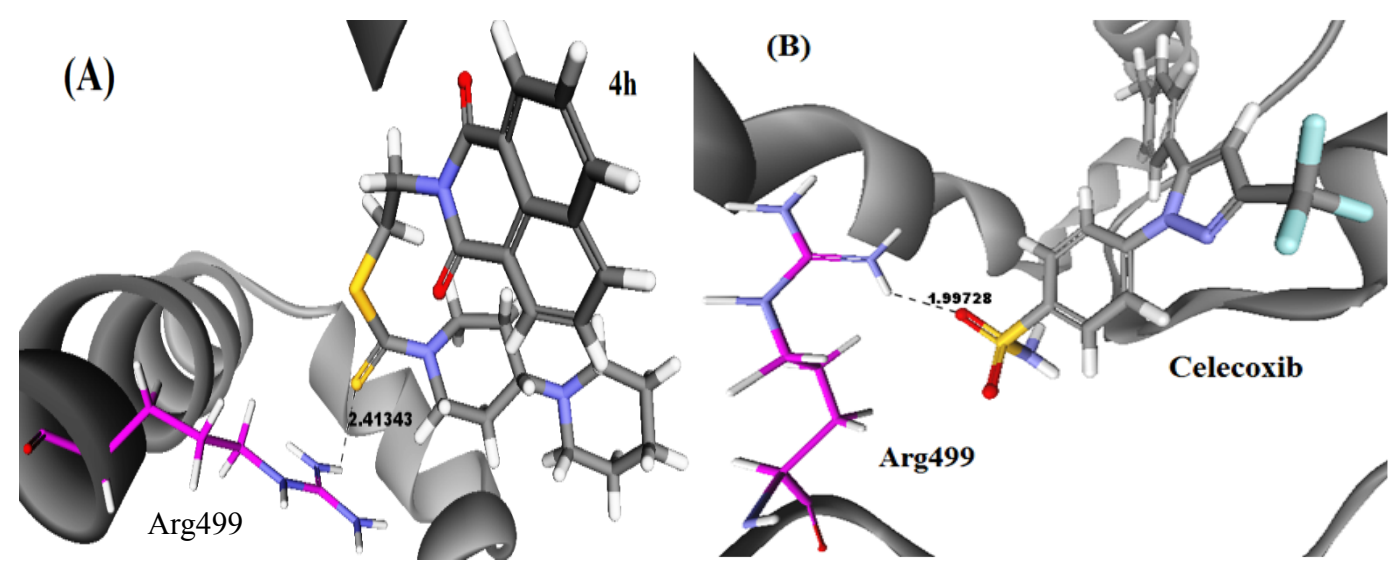

Figure 2: Docking view of 4d (A) and Celecoxib (B) in the binding site of COX-2 (pdb code 3LN1), forming $1 \mathrm{H}$-bond with Arg499 with distance $2.41 \mathrm{~A}^{\circ}$, and $1.99 \mathrm{~A}^{\circ}$, respectively.

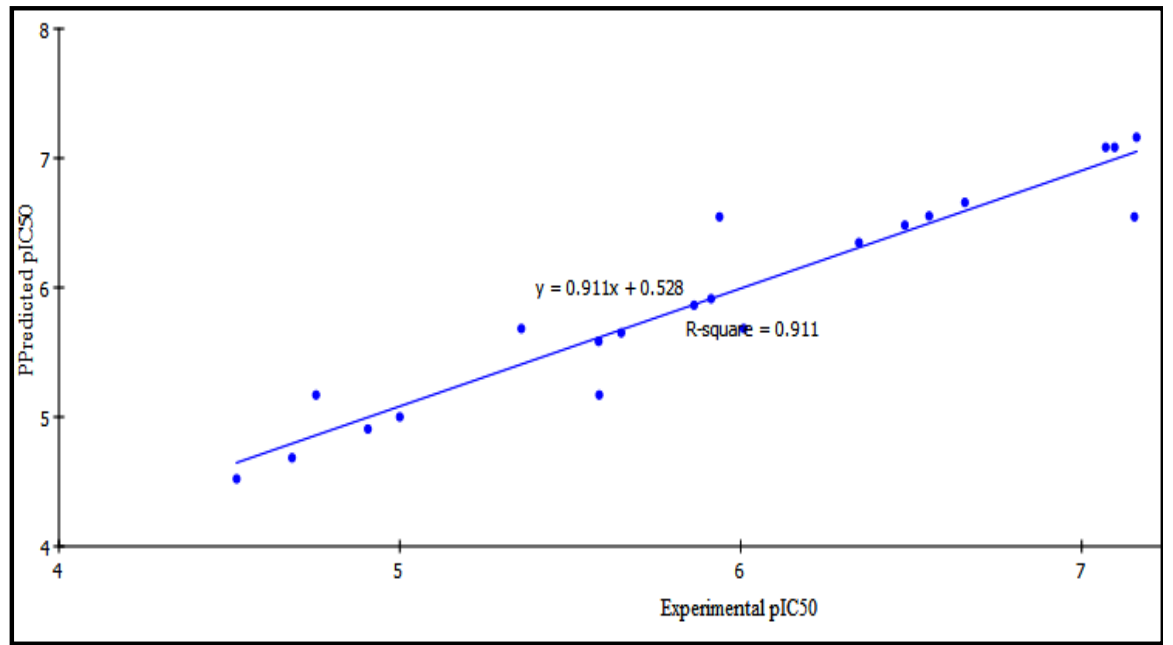

Figure 3: The corresponding scatter plots of the experimental versus the predicted bioactivity values plC $_{50}$ for the training set compounds according to Equation 1 $\left(r^{2}=0.911\right)$. 
Citation: Zahra M, Osman A, Agwa H, Nair N, Sanchez A, et al. (2016) Synthesis, Biological Evaluation, Docking and QSAR Studies of Some Novel Naphthalimide Dithiocarbamate Analogs as Antitumor and Anti-Inflammatory Agents. Med Chem (Los Angeles) 6: 694-703. doi:10.4172/2161-0444.1000417

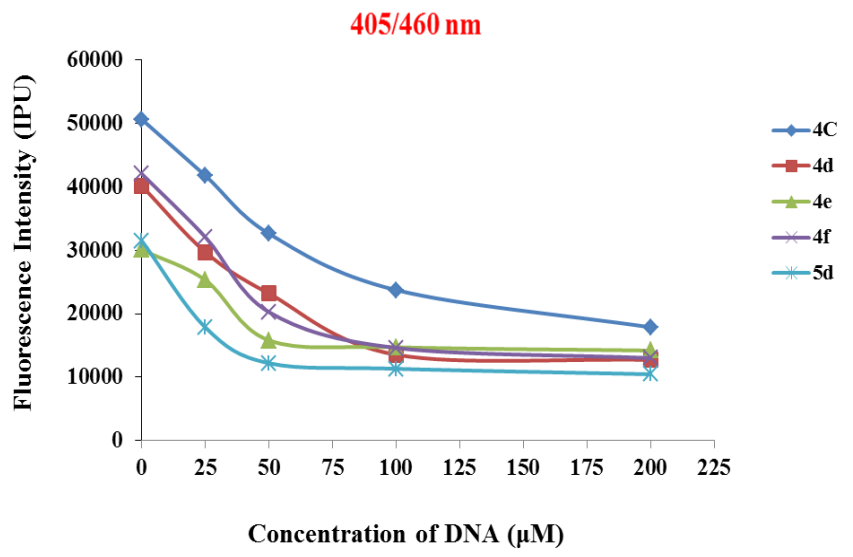

Figure 4: Fluorescence spectrum after interaction of naphthalimide analogs with calf thymus DNA.

\begin{tabular}{|c|c|c|c|c|}
\hline \multirow{2}{*}{ Compound } & \multicolumn{2}{|c|}{ COX-2 (pdb code 3LN1) } & \multicolumn{2}{|c|}{ COX-1 (pdb code $3 K K 6$ ) } \\
\hline & -CDOCKER energy & H-bonds & -CDOCKER energy & H-bonds \\
\hline Celecoxib & 46.87 & 1- with Arg499 & 13.76 & 2- with Tyr355 \\
\hline 4c & 49.01 & 1- with Arg499 & 15.32 & No H-bonds \\
\hline 4d & 54.23 & 1-with Arg499 & 15.62 & 1- with Arg120 \\
\hline $4 e$ & 45.37 & 1- with Arg499 & 13.01 & 3 - with GIn192, Ile517 and Ser530 \\
\hline $4 f$ & 47.08 & 1- with His75 & 13.34 & 1- with Ser530 \\
\hline $5 d$ & 44.15 & 1-with His75 & 12.79 & No H-bonds \\
\hline
\end{tabular}

Table 4: Docking results for the synthesized compounds.

\begin{tabular}{|c|c|c|c|}
\hline Compound & Experimental plC $\mathrm{P}_{50}$ & Predicted $\mathrm{plC}_{50}$ & Residuals \\
\hline 4c & 6.13077 & 7.3086 & 1.17783 \\
\hline $4 d$ & 6.26761 & 7.4219 & 1.15429 \\
\hline $4 e$ & 5.70333 & 6.55244 & 0.84911 \\
\hline $4 f$ & 5.84771 & 6.51472 & 0.66701 \\
\hline $5 d$ & 5.49894 & 6.97452 & 1.47558 \\
\hline Celecoxib a & 5.91010 & 6.69213 & 0.78203 \\
\hline
\end{tabular}

Table 5: Experimental activity of the synthesized compounds against the predicted activity according to Equation $1^{\text {a }}$.

naphtalimides analogues with flexible side chains (dithiocarbamate as well as dithioate). In this study compound $4 \mathbf{d}$ showed a broad spectrum antitumor activity and exhibited potent anti-inflammatory effect; the experimental as well as the theoretical studies clearly predicts that bipiperidine group is essential for the activities. Moreover the result; revealed the direct correlation between the COX inhibition and anticancer activity. In addition to derivative $4 c$, with amino alkyl chain; inhibited the growth of SKBR-3 by $84.13 \%\left(\mathrm{IC}_{50}: 10.54 \mu \mathrm{M}\right)$; this analogue could be used as a promising target for future development of new anti-breast agents. Further investigation to gain more insight the molecular mechanism is currently under planning in the Laboratory of Nano-Biotechnology, Department of Medical Bioengineering Science, Okayama University, Kita-ku, Okayama, Japan.

\section{Acknowledgements}

The authors would like to thank Prof. Erik B. Pedersen; Department of Physics, Chemistry and Pharmacy, Nucleic Acid Center, University of Southern Denmark, Odense, Denmark. This study was partly supported by MEXT/JSPS
KAKENHI Grant Numbers 26640079 and 25242045 also Science and Technology Development Fund (STDF) through project No. 3627.

\section{References}

1. Waghorne CL, Corkran HM, Hunt-Painter AA, Niktab E, Baty JW, et al. (2016) $\mathrm{N}, \mathrm{N}-\mathrm{Bis}$ (glycityl)amines as anti-cancer drugs. Bioorg Med Chem 24: 3932 3939 .

2. Chan TA (2002) Nonsteroidal anti-inflammatory drugs, apoptosis, and coloncancer chemoprevention. The lancet oncology 3: 166-174.

3. Gasparini G, Longo R, Sarmiento R, Morabito A (2003) Inhibitors of cyclooxygenase 2: a new class of anticancer agents. Lancet Oncology 4: 605-615.

4. Ott I, Xu Y, Liu J, Kokoschka M, Harlos M, et al. (2008) Sulfur-substituted naphthalimides as photoactivatable anticancer agents: DNA interaction, fluorescence imaging, and phototoxic effects in cultured tumor cells. Bioorganic \& medicinal chemistry 16: 7107-7116.

5. Braña MF, Cacho M, Gradillas A, de Pascual-Teresa B, Ramos A (2001) Intercalators as anticancer drugs. Curr Pharm Des 7: 1745-1780. 
Citation: Zahra M, Osman A, Agwa H, Nair N, Sanchez A, et al. (2016) Synthesis, Biological Evaluation, Docking and QSAR Studies of Some Novel Naphthalimide Dithiocarbamate Analogs as Antitumor and Anti-Inflammatory Agents. Med Chem (Los Angeles) 6: 694-703. doi:10.4172/2161-0444.1000417

6. Hargreaves K, Pritchard JG, Dave HR (1970) cyclic carboxylic monoimides. Chemical Review 70: 439-469.

7. Andricopulo AD, Willain-Filho A, Corrêa R, Santos AR, Nunes RJ, et al. (1998) Analgesic activity of 3, 4-dichloromaleimides: structure-activity relationships. Die Pharmazie 53: 493-494

8. Cos P, Calomme M, Sindambiwe JB, De Bruyne T, Cimanga K, et al. (2001) Cytotoxicity and lipid peroxidation-inhibiting activity of flavonoids. Planta Med 67: 515-519.

9. Cechinel Filho V, Bella Cruz A, Moretto E, Pinheiro T, Nunes RJ, et al. (1994) Antibacterial activity of $\mathrm{N}$-phenylmaleimides, $\mathrm{N}$-phenylsuccinimides and related compounds; Structure-activity relationships. Farmaco 49: 675-677.

10. Antonini I, Volpini R, Ben DD, Lambertucci C, Cristalli G (2008) Design, synthesis, and biological evaluation of new mitonafide derivatives as potential antitumor drugs. Bioorganic Medicinal Chemistry 16: 8440-8446.

11. Matsubayashi K, Shiratori $\mathrm{H}$, Kubo $\mathrm{Y}$ (2010) Effect of addition of trifluoroacetic acid on the photophysical properties and photoreactions of aromatic imides. Tertahedron 66: 9291-9299.

12. Li Z, Yang Q, Qian X (2005) Novel heterocyclic family of phenyl naphthothiazole carboxamides derived from naphthalimides: synthesis, antitumor evaluation, and DNA photocleavage. Bioorganic Medicinal Chemistry 13: 3149-3155.

13. Chen Z, Liang X, Zhang H, Xie H, Liu J, et al. (2010) A new class of naphthalimide-based antitumor agents that inhibit topoisomerase II and induce lysosomal membrane permeabilization and apoptosis. Journal of Medicinal Chemistry 53: 2589-2600

14. Ott I, Qian X, Xu Y, Vlecken DHW, Marques IJ, et al. (2009) Gold(I) Phosphine Complex Containing a Naphthalimide Ligand Functions as a TrxR Inhibiting Antiproliferative Agent and Angiogenesis Inhibitor. Journal of Medicinal Chemistry 52: 763-770.

15. Ott I, Xu Y, Qian X (2011) Fluorescence properties and antiproliferative effects of mono-, bis-, and tris- thiophenylnaphthalimides: results of a comparative pilot study. J Photochem Photobiol B 105: 75-80.

16. Kamal A, Ramu R, Tekumalla V, Khanna GBR, Barkume MS, et al. (2008) Remarkable DNA binding affinity and potential anticancer activity of pyrrolo[2,1-c][1,4]benzodiazepine-naphthalimide conjugates linked through piperazine side-armed alkane spacers. Bioorganic Medicinal Chemistry 16 7218-7224.

17. Qian X, Li Y, Xu Y, Qu B (2004) Highly-efficient DNA photocleavers with long wavelength absorptions: thio-heterocyclic fused naphthalimides containing aminoalkyl side chains. Bioorganic Medicinal Chemistry Letter 14: 2665-2668.

18. Li Y, Xu Y, Qian X, Qu B (2004) Naphthalimide-thiazoles as nove photonucleases: molecular design, synthesis, and evaluation. Tetrahedron Letter 45: 1247-1251.

19. Verma M, Luxami V, Paul K (2013) Synthesis, in vitro evaluation and molecular modelling of naphthalimide analogue as anticancer agents. Eur J Med Chem 68: $352-360$

20. Li Z, Yang Q, Qian X (2005) Novel thiazonaphthalimides as efficient antitumor and DNA photocleaving agents: effects of intercalation, side chains, and substituent groups. Bioorganic Medicinal Chemistry 13: 4864-4870.

21. Len C, Boulogne-merlot AS, Postel D, Ronco G, Villa P, et al. (1996) Synthesis and antifungal activity of novel bis(dithiocarbamate) derivatives of glycerol. Journal of Agriculture Food and Chemistry 44: 2856-2858.

22. Imamura $H$, Ohtake $N$, Jona $H$, Shimizu A, Moriya M, et al. (2001) Dicationic dithiocarbamate carbapenems with anti-MRSA activity. Bioorg Med Chem 9: 1571-1578.

23. Carta F, Aggarwal M, Maresca A, Scozzafava A, McKenna R, et al. (2012) Dithiocarbamates strongly inhibit carbonic anhydrases and show antiglaucoma action in vivo. J Med Chem 55: 1721-1730.

24. Carta F, Supuran CT (2012) Dithiocarbamates: a new class of carbonic anhydrase inhibitors crystallographic and kinetic investigations. Chemical Communication 48: 1868-1870.
25. Huang W, Ding Y, Miao Y, Liu MZ, Li Y, et al. (2009) Synthesis and antitumor activity of novel dithiocarbamate substituted chromones. European Journal of Medicinal Chemistry 44: 3687-3696.

26. Brahemi G, Kona FR, Fiasella A, Buac D, Soukupova J, et al. (2010) Exploring the structural requirements for inhibition of the ubiquitin E3 ligase breast cancer associated protein 2 (BCA2) as a treatment for breast cancer. Journal of Medicinal Chemistry 53: 2757-2765

27. Zahran MAH, Salem TAR, Samaka RM, Agwa HS, Awad AR (2008) Design synthesis and antitumor evaluation of novel thalidomide dithiocarbamate and dithioate analogs against Ehrlich ascites carcinoma-induced solid tumor in Swiss albino mice. Bioorganic Medicinal Chemistry 16: 9708-9718.

28. Guirgis AA, Zahran MAH, Mohamed AS, Talaat RM, Abdou BY et al. (2010) Effect of thalidomide dithiocarbamate analogs on the intercellular adhesion molecule-1 expression. International Immunopharmacology 10: 805-811.

29. Xie L, Xu Y, Wang F, Liu J, Qian X, et al. (2009) Synthesis of new amonafide analogues via coupling reaction and their cytotoxic evaluation and DNA-binding studies. Bioorganic Medicinal Chemistry 17: 804-810.

30. Winter CA, Risley EA, Nuss GW (1962) Carrageenin-induced edema in hind paw of the rat as an assay for antiiflammatory drugs. Proc Soc Exp Biol Med 111: 544-547.

31. Talaat R, El-Sayed W, Agwa H, Gamal-Eldeen A, Moawia S, et al. (2015) AntiInflammatory Effect of Thalidomide Dithiocarbamate and Dithioate Analogs. Journal of Chemico-Biological Interaction 238: 74-81.

32. Zahran MA, Abdin YG, Osman AM, Gamal-Eldeen AM, Talaat RM, et al. (2014) Synthesis and evaluation of thalidomide and phthalimide esters as antitumor agents. Arch Pharm (Weinheim) 347: 642-649.

33. Hossain SU, Sengupta S, Bhattacharya S (2005) Synthesis and evaluation of antioxidative properties of a series of organoselenium compounds. Bioorganic Medicinal Chemistry 13: 5750-5758

34. Asama M, Suva H, Sushanta D, Shanmugavel M, Manikrao MD, et al. (2011) Antitumor efficacy and apoptotic activity of substituted chloroalkyl $1 \mathrm{H}$-benz[de] isoquinoline-1,3-diones: a new class of potential antineoplastic agents. Investigational New Drugs 29: 434-442.

35. Alexander HS, Igor VS, Julia AW, Michael DW (2012) Controllable threecomponent luminescence from a 1,8-naphthalimide/Eu(III) complex: white light emission from a single molecule. Chemical Communication 48: 2749-2751.

36. Xiao S, Lin W, Wang C, Yang M (2001) Synthesis and biological evaluation of DNA targeting flexible side-chain substituted beta-carboline derivatives. Bioorganic Medicinal Chemistry Letter 11: 437-441.

37. Xie L, Qian X, Cui J, Xiao Y, Wang K, et al. (2008) Novel angula furoquinolinones bearing flexible chain as antitumor agent: design, synthesis cytotoxic evaluation, and DNA-binding studies. Bioorganic Medicinal Chemistry 16: $8713-8718$

38. Guan H, Chen H, Peng W, Ma Y, Cao R, et al. (2006) Design of ß-carboline derivatives as DNA-targeting antitumor agents. European Journal of Medicinal Chemistry 41: 1167-1179.

39. Li Z, Yang Q, Qian X (2005) Synthesis, antitumor evaluation and DNA photocleaving activity of novel methylthiazonaphthalimides with aminoalky side chains. Bioorganic Medicinal Chemistry Letter 13: 3143-3146.

40. Shigehiro T, Zhai W, Vaidyanath A, Seno M, Kasai T, et al. (2016) Evaluation of glycosylated docetaxel-encapsulated liposomes prepared by remote loading under solubility gradient. Journal of Microencapsulation 1-11.

41. Feng L, Jingnan C, Lianying G, Xuhong Q, Weimin R, et al. (2007) Molecular design, chemical synthesis, and biological evaluation of '4-1' pentacyclic aryl/ heteroaryl-imidazonaphthalimides. Bioorganic Medicinal Chemistry 15: 5114 5121.

42. Pommery N, Taverne T, Telliez A, Goossens L, Charlier C, et al. (2004) New COX-2/5-LOX Inhibitors:? Apoptosis-Inducing Agents Potentially Useful in Prostate Cancer Chemotherapy. Journal of Medicinal Chemistry 47: 6195-6206

43. Khan MN, Lee YS (2011) Cyclooxygenase inhibitors: scope of their use and development in cancer chemotherapy. Medicinal Research Reviews 31: 161-201 
Citation: Zahra M, Osman A, Agwa H, Nair N, Sanchez A, et al. (2016) Synthesis, Biological Evaluation, Docking and QSAR Studies of Some Novel Naphthalimide Dithiocarbamate Analogs as Antitumor and Anti-Inflammatory Agents. Med Chem (Los Angeles) 6: 694-703. doi:10.4172/2161-0444.1000417

44. Boland GP, Butt IS, Prasad R, Knox WF, Bundred NJ (2004) COX-2 expression is associated with an aggressive phenotype in ductal carcinoma in situ. British Journal of Cancer 90: 423-429.

45. Wu G, Robertson DH, Brooks CL 3rd, Vieth M (2003) Detailed analysis of gridbased molecular docking: A case study of CDOCKER-A CHARMm-based MD docking algorithm. J Comput Chem 24: 1549-1562.

46. Zarghi A, Ghodsi R, Azizi E, Daraie B, Hedayati M, et al. (2009) Synthesis and biological evaluation of new 4-carboxyl quinoline derivatives as cyclooxygenase-2 inhibitors. Bioorganic Medicinal Chemistry 17: 5312-5317

47. Zarghi A, Zebardast T, Daraie B, Hedayati M (2009) Design and synthesis of new 1,3-benzthiazinan-4-one derivatives as selective (COX-2) inhibitors. Bioorganic Medicinal Chemistry 17: 5369-5373.

48. PIsikova J, Janovec L, Koval J, Ungvarsky J, Mikes J, et al. (2012) 3,6-bis(3alkylguanidino)acridines as DNA-intercalating antitumor agents. Eur $\mathrm{J}$ Med Chem 57: 283-295. 